\title{
Performance Evaluation of Two-Hop Wireless Network under Asymmetric Fading Environment
}

\author{
Sarwar Jahan1, Md Imdadul Islam², Mohamed Ruhul Amin1 \\ ${ }^{1}$ Department of Electronics and Communication Engineering, East West University, Dhaka, Bangladesh \\ ${ }^{2}$ Department of Computer Science and Engineering, Jahangirnagar University, Savar, Bangladesh \\ Email: ramin@ewubd.edu
}

Received 29 October 2015; accepted 4 December 2015; published 8 December 2015

Copyright (C) 2015 by authors and Scientific Research Publishing Inc.

This work is licensed under the Creative Commons Attribution International License (CC BY). http://creativecommons.org/licenses/by/4.0/

c) (7) Open Access

\begin{abstract}
To enhance the reliability of long wireless link, a relay is placed between transmitter and receiver which break the link into two parts: transmitter to relay called $1^{\text {st }}$ hop and relay to receiver called $2^{\text {nd }}$ hop; such arrangement is called two hop wireless links. In two-hop wireless communication sometimes $1^{\text {st }}$ and $2^{\text {nd }}$ hops are affected by different types of fading called asymmetric fading environment. In this paper we consider four different combinations of Rayleigh and Nakagami-m fading on $1^{\text {st }}$ and $2^{\text {nd }}$ hop to observe the profile of combined pdf (probability density function) and cdf (cumulative distribution function). With the help of cdf we determine the variation of outage probability of mixed fading. Finally we plot the SER (symbol error rate) against the SNR (signal to noise ratio) of the $2^{\text {nd }}$ hop for all possible combinations of the two fading taking the SNR of $1^{\text {st }}$ hop as a parameter. Although recent literature deals with asymmetric fading environment but in this paper instead of details statistical model of such fading we provide the relative scenario of mixed fading on 2-hop wireless link.
\end{abstract}

\section{Keywords}

Two-Hop, Asymmetric Network, Relay Network, Rayleigh Fading, Nakagami Fading

\section{Introduction}

Performance of wireless communication suffers from different fading effects and these effects can be reduced by using multiple-input multiple-output (MIMO), or relayed transmission technique. Consequently, this leads to a great research interest in the MIMO and the relayed communications techniques. In amplify-and-forward (AF) 
relayed transmission technique, a wireless link has been established between the source and the destination via the relay. Here the source is transmitting a signal to the relay (known as $1^{\text {st }}$ hop S-R) and relay detects the signal with amplification sending it to the destination (known as $2^{\text {nd }}$ hop R-D) [1]. On the accessibility of instantaneous channel sate information (CSI) at the relay node, AF relaying scheme is generally categorized into two parts, one is variable-gain relaying [2] and the other is fixed gain AF relaying [3]. In such communication networks, the signal may be affected by different types of fading. Taking the effect of fading in account, we can divide AF relay network into two categories, one is for symmetric fading channels and the other is for asymmetric fading channels. In the past years, the performance of relay networks (two ways, dual hope, multi hope, and multi cast) over symmetric fading channels is being carried out by many researchers [4]. The performances of dual hop wireless link under symmetric (Nakagami-m) fading channels have been discussed in [5]. However, in practical scenario, relay network may have line of sight (LOS) communication from source to destination and multipath communication from relay to destination and vice versa.

In present days the research interest on relay network over asymmetric fading channels has been increased and some recent examples of that are [6]-[8]. For dual hop AF relay transmission system, the performance of the network has been studied in [7] by deriving analytical expression for the cumulative distribution function (cdf) and the probability density function (pdf) of end to end signal to noise ratio (SNR) over asymmetric (Nakagami-m and Rician) fading channels. Here authors have studied the performance of beam forming in dual hop AF network and have derived closed-form cdf, pdf and expressions of the received SNR and average symbol error rate (SER) for Rayleigh fading channel. The outage probability and the average bit error probability have been studied in [8] for asymmetric (Rayleigh and Rician) fading channels. In both cases ergodic capacity analysis has been taken into consideration. In [9], closed-form cdf, pdf and moment generating function (MGF), expressions of received SNR and SER have been derived for MIMO AF relay network over a Rayleigh fading channel, where transmit antenna selection and maximal ratio combining are used at the source and the destination, respectively, and the relay is equipped with a single antenna [10]. In such an asymmetric fading scenario, the above studies are promising for improving the performance of a wireless communication link.

In this paper, we assume dual hope AF relay network over symmetric and asymmetric fading channels and study the performance of the relay network by evaluating the closed form expressions for outage probability, average SER by using cdf and ergodic capacity by using pdf. We plot the analytical results for the variation of combined cdf and pdf against the instantaneous SNR considering different symmetric (Nakagami-Nakagami, Releigh-Rayleigh) and asymmetric (Nakagami-m-Rayleigh, Rayleigh-Nakagami-m) fading channels for a relay network. Finally, the effects of these fading channels on the relay network are shown by considering the variation of SER against SNR of the $2^{\text {nd }}$ hop. It is to be mentioned here that in two-hop wireless link, there is a good possibility of experiencing different fading environments in first and second hop. Most of the papers claim their contribution in deriving the expression of SER and Outage probability in closed forms from different angles, where the expressions become very complicated. Here, we are only interested to find the solution in a simpler method.

The paper is organized as follows: Section 2 provides the theoretical analysis in determination of the combined pdf and cdf of 2-hop wireless link along with SER, Section 3 deals with the results based on analysis of Section 2 and finally Section 4 concludes the entire analysis.

\section{Methodology}

In a 2-hop wireless link sometimes the $1^{\text {st }}$ hop (S-R) and the $2^{\text {nd }}$ hop (R-D) experience different types of fading called asymmetric or mixed fading environment as discussed in [4] [5] [7] [8] and [11]. Let the pdf of the SNR of $1^{\text {st }}$ hop is $f_{\Gamma_{1}}\left(\gamma_{1}, \Omega\right)$ and that of $2^{\text {nd }}$ hop is $f_{\Gamma_{2}}\left(\gamma_{2}, \gamma_{a v}\right)$; where $\Omega$ and $\gamma_{a v}$ are the average SNRs of the corresponding links. The cdf of the combined link is expressed [11]:

$$
F_{\Gamma}(\tau)=\int_{0}^{\infty} \operatorname{Pr}\left\{\gamma_{e q}<\tau \mid \gamma_{2}\right\} f_{\Gamma_{2}}\left(\gamma_{2}\right) \mathrm{d} \gamma_{2},
$$

where

$$
\gamma_{e q}=\frac{\gamma_{1} \gamma_{2}}{\gamma_{1}+\gamma_{2}+c}
$$


and

$$
\gamma_{e q}<\tau \Rightarrow \frac{\gamma_{1} \gamma_{2}}{\gamma_{1}+\gamma_{2}+c}<\tau \Rightarrow \gamma_{1}<\frac{\left(\gamma_{2}+c\right) \tau}{\gamma_{2}-\tau}=\gamma^{\prime} .
$$

Equation (1) can then be written as

$$
F_{\Gamma}(\tau)=\int_{0}^{\infty} \operatorname{Pr}\left\{\gamma_{1}<\gamma^{\prime} \mid \gamma_{2}\right\} f_{\Gamma_{2}}\left(\gamma_{2}\right) \mathrm{d} \gamma_{2}=\int_{0}^{\infty} F_{\Gamma_{1}}\left(\gamma^{\prime}\right) f_{\Gamma_{2}}\left(\gamma_{2}\right) \mathrm{d} \gamma_{2}
$$

where $F_{\Gamma_{1}}\left(\gamma^{\prime}\right)$ is the cdf of $1^{\text {st }}$ link.

If S-R link is under Nakagami- $m$ fading then its pdf

$$
f_{\Gamma_{1}}\left(\gamma_{1}, \Omega\right)=\left(\frac{m}{\Omega}\right)^{m} \frac{\gamma_{1}^{m-1}}{\Gamma(m)} \mathrm{e}^{-\frac{m}{\Omega} \gamma_{1}} .
$$

Based on [4] [7] [8] the cdf will be,

$$
F_{\Gamma_{1}}\left(\gamma^{\prime}\right)=\operatorname{Pr}\left\{\gamma_{1} \leq \gamma^{\prime}\right\}=1-\frac{\Gamma\left(m,\left(\frac{m}{\Omega}\right) \gamma^{\prime}\right)}{\Gamma(m)}=1-\frac{\Gamma\left(m,\left(\frac{m}{\Omega}\right)\left(\frac{\gamma_{2}+c}{\gamma_{2}-\tau}\right) \tau\right)}{\Gamma(m)}=F_{\Gamma_{1}}(\tau) .
$$

From Equations (2) and (4),

$$
\begin{aligned}
F_{\Gamma}(\tau) & =\int_{0}^{\infty}\left\{1-\frac{\Gamma\left(m,\left(\frac{m}{\Omega}\right)\left(\frac{\gamma_{2}+c}{\gamma_{2}-\tau}\right) \tau\right)}{\Gamma(m)}\right\} f_{\Gamma_{2}}\left(\gamma_{2}\right) \mathrm{d} \gamma_{2} \\
& =1-\int_{0}^{\infty} \frac{\Gamma\left(m,\left(\frac{m}{\Omega}\right)\left(\frac{\gamma_{2}+c}{\gamma_{2}-\tau}\right) \tau\right)}{\Gamma(m)} f_{\Gamma_{2}}\left(\gamma_{2}\right) \mathrm{d} \gamma_{2}
\end{aligned}
$$

If the R-D link is under Rayleigh fading then,

$$
f_{\Gamma_{2}}\left(\gamma_{2}\right)=\frac{1}{\gamma_{a v}} \mathrm{e}^{-\gamma_{2} / \gamma_{a v}} .
$$

From Equations (5) and (6), we have

$$
F_{\Gamma}(\tau)=1-\int_{0}^{\infty} \frac{\Gamma\left(m,\left(\frac{m}{\Omega}\right)\left(\frac{\gamma_{2}+c}{\gamma_{2}-\tau}\right) \tau\right)}{\Gamma(m)} \frac{1}{\gamma_{a v}} \mathrm{e}^{-\gamma_{2} / \gamma_{a v}} \mathrm{~d} \gamma_{2} .
$$

From [5], for the condition $\gamma_{2}>\tau$, the range of $\gamma_{2}$ will be $[\gamma, \infty]$. Again for the condition, $\gamma_{2}<\tau$, the range of $\gamma_{2}$ will be $[0, \gamma]$. Hence, Equations (4) to (7) has to be changed accordingly.

Putting $\tau$ is equal to threshold SNR $\gamma_{t h}$ in Equation (7) we get the outage probability. Differentiating Equation (7), we get the pdf of the combined link as

$$
f_{\Gamma}(\gamma)=\frac{\mathrm{d} F_{\Gamma}(\gamma)}{\mathrm{d} \gamma} .
$$

The BER can be determined according to [12] as

$$
P_{b}\left(\Omega, \gamma_{a v}\right)=\int_{0}^{\infty} Q(\sqrt{\beta \gamma}) f_{\Gamma}(\gamma) \mathrm{d} \gamma,
$$

where the magnitude of $\beta$ depends on the particular modulation scheme (for example $\beta=1$ for QPSK, 
$\beta=2$ for BPSK, etc.).

The $2^{\text {nd }}$ formula for SER is

$$
P_{s}\left(\Omega, \gamma_{a v}\right)=\int_{0}^{\infty} a Q(\sqrt{2 b \gamma}) f_{\Gamma}(\gamma) \mathrm{d} \gamma
$$

The $3^{\text {rd }}$ formula for SER [13] is

$$
P_{s}\left(\Omega, \gamma_{a v}\right)=\frac{a \sqrt{b}}{2 \sqrt{\pi}} \int_{0}^{\infty} \frac{\mathrm{e}^{-b \gamma}}{\sqrt{\gamma}} F_{\Gamma}(\gamma) \mathrm{d} \gamma
$$

It is to be mentioned here that, for M-ary-QAM $b=1.5 /(M-1)$ and $a=4(\sqrt{M}-1) / \sqrt{M}$.

\section{Results}

The variation of combined cdf and pdf against the instantaneous SNR are shown in Figures 1-4 (four combinations of fading) taking average SNR (absolute) of the $1^{\text {st }}$ hop and the $2^{\text {nd }}$ hop as a parameter. Both cdf and pdf are found stepper for lower values of SNR-1 and SNR-2 for all the four combinations of fading. The profile of pdf and cdf are all exponential but the pdf of Nakagami-Nakagami and Nakagami-Rayleigh combinations at higher average SNR provides pdf like Poisson's pdf is shown in Figure 2 and Figure 3. With the help of both pdf and cdf, we determine the SER of 2-hop link with mixed fading under QPSK. The variation of SER against average SNR of $2^{\text {nd }}$ hop is shown in Figure 5 taking average SNR of $1^{\text {st }}$ hop of $5 \mathrm{~dB}, m_{1}=2$, and $m_{2}=4$. The performance is found the best for Nakagami-Nakagami combination and worst for Rayleigh-Rayleigh combination. In Nakagami- $m$ fading, there are $m$ direct link between transmitter and receiver hence the results of Figure 5 is obvious. We also include the SER of single hop link under both Rayleigh and Nakagami- $m$ fading case in Figure 5 to get the idea about the amount degradation of 2-hop link for 4 possible fading combinations. We have to allude one thing that if the length of the single hop link is equal or greater that the 2-hop link then the performance of single hop will be much worse than the case of 2-hop link. Our next step is to make the $1^{\text {st }}$ hop stronger taking SNR of $1^{\text {st }}$ hop of $12 \mathrm{~dB}, m_{1}=2$, and $m_{2}=4$. The corresponding profile of SER is shown in Figure 6. This time the performance of the network is found much better than the case of Figure 5. In this case stronger signal will be received by the relay hence the received signal will maintain SNR greater than the threshold. Therefore, the impact of pdf of $1^{\text {st }}$ hop is more prominent than that of $2^{\text {nd }}$ hop.

Finally, the performance of mixed 2-hop ( $1^{\text {st }}$ hop is Nakagami- $m, m=4$ and $2^{\text {nd }}$ hop is Rayleigh) wireless link is shown in Figure 7 in context of outage probability vs. average SNR of $2^{\text {nd }}$ hop. The performance of the communication system enhances with increments of average SNR of $2^{\text {nd }}$ hop and with the decrement of the threshold SNR of $1^{\text {st }}$ hop is visualized from Figure 7.

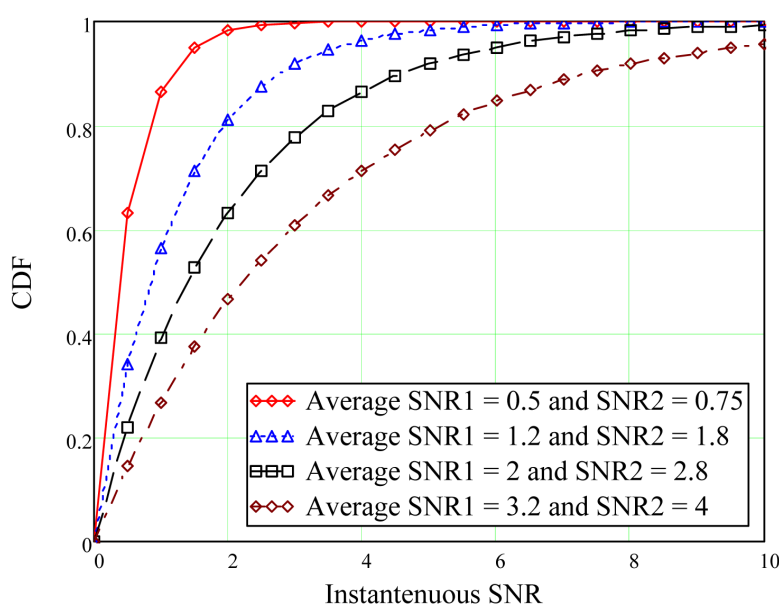

(a)

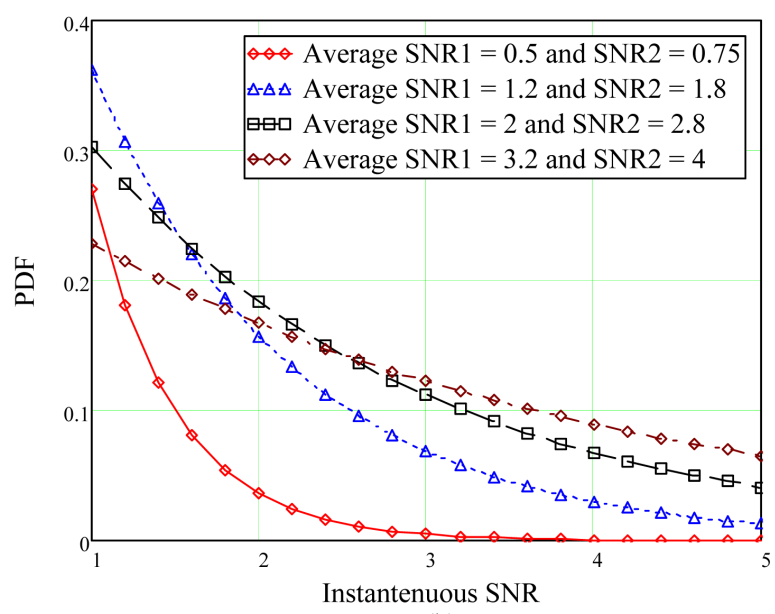

(b)

Figure 1. CDF and PDF of combined Rayleigh-Rayleigh fading case. (a) CDF of 2-hop link; (b) PDF of 2-hop link. 


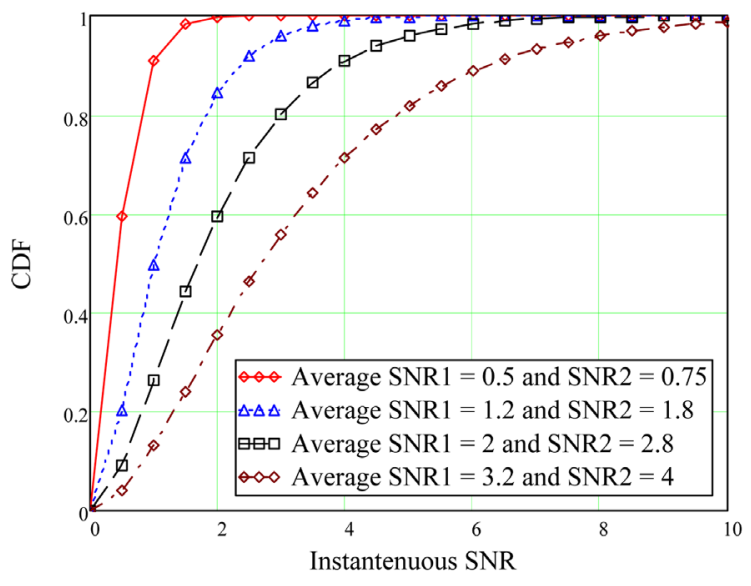

(a)

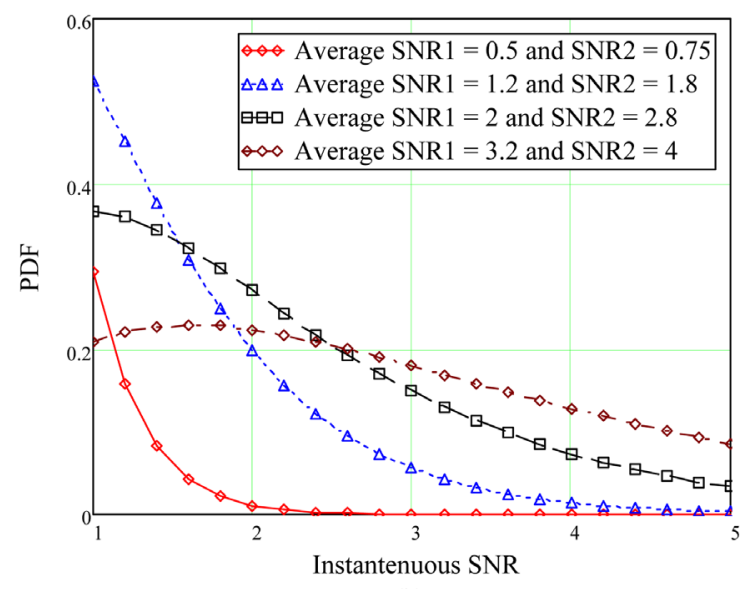

(b)

Figure 2. CDF and PDF of combined Nakagami-Nakagami fading case $\left(m_{1}=2, m_{2}=4\right)$. (a) CDF of 2-hop link; (b) PDF of 2-hop link.

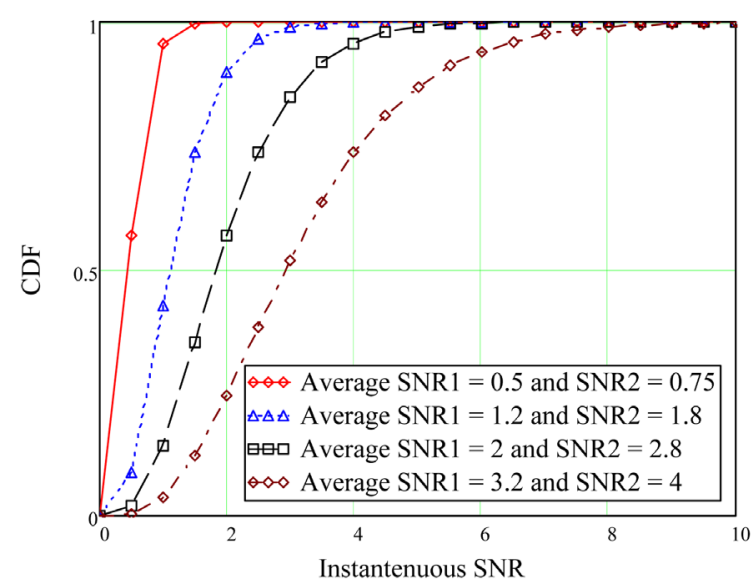

(a)

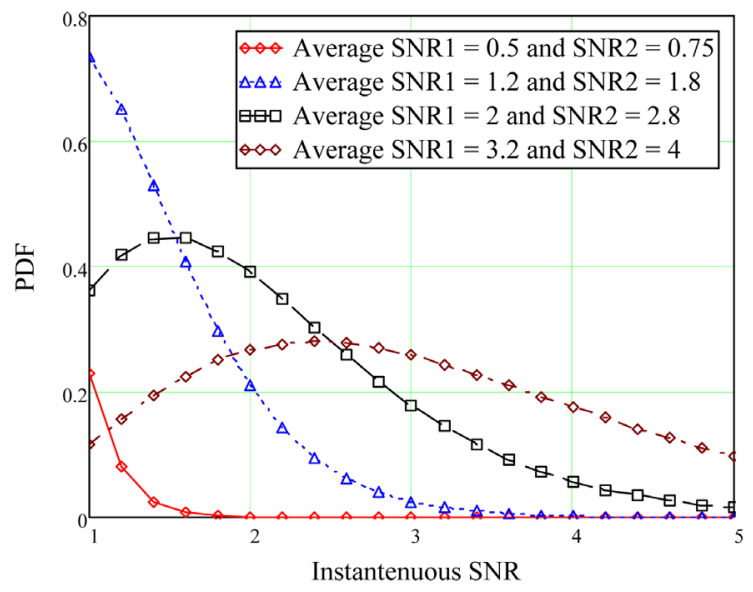

(b)

Figure 3. CDF and PDF of mixed (Nakagami-m and Rayleigh) fading case $\left(m_{1}=4\right)$. (a) CDF of 2-hop link; (b) PDF of 2-hop link.

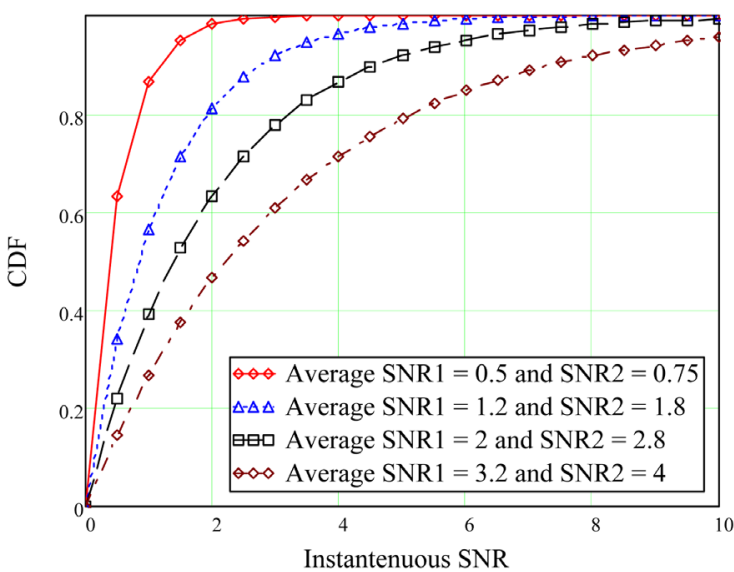

(a)

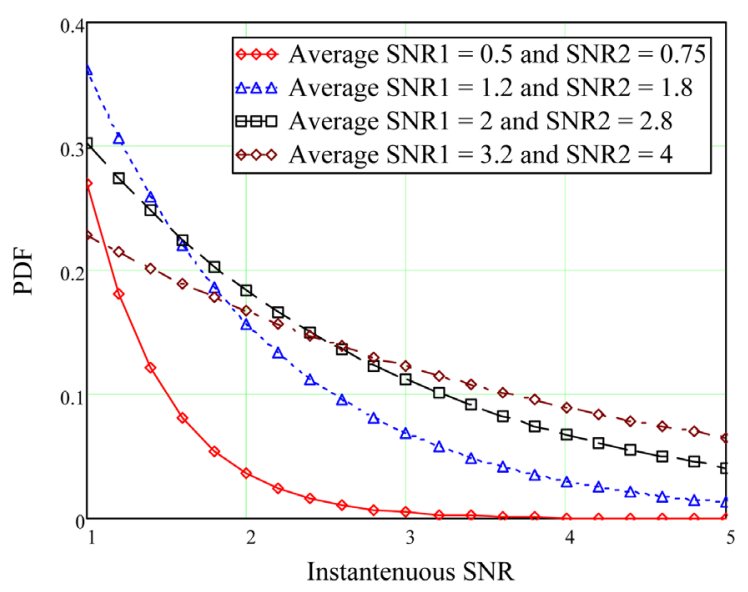

(b)

Figure 4. CDF and PDF of mixed (Rayleigh and Nakagami-m) fading case $\left(m_{2}=4\right)$. (a) CDF of 2-hop link; (b) PDF of 2-hop link. 


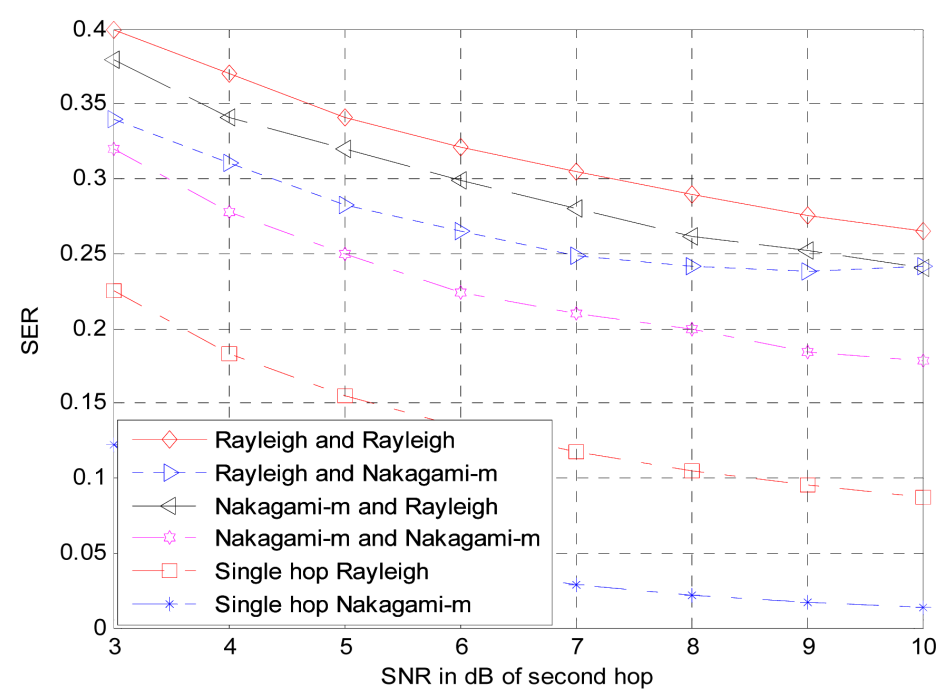

Figure 5. variation of SER against SNR of $2^{\text {nd }}$ hop (weak $1^{\text {st }}$ hop).

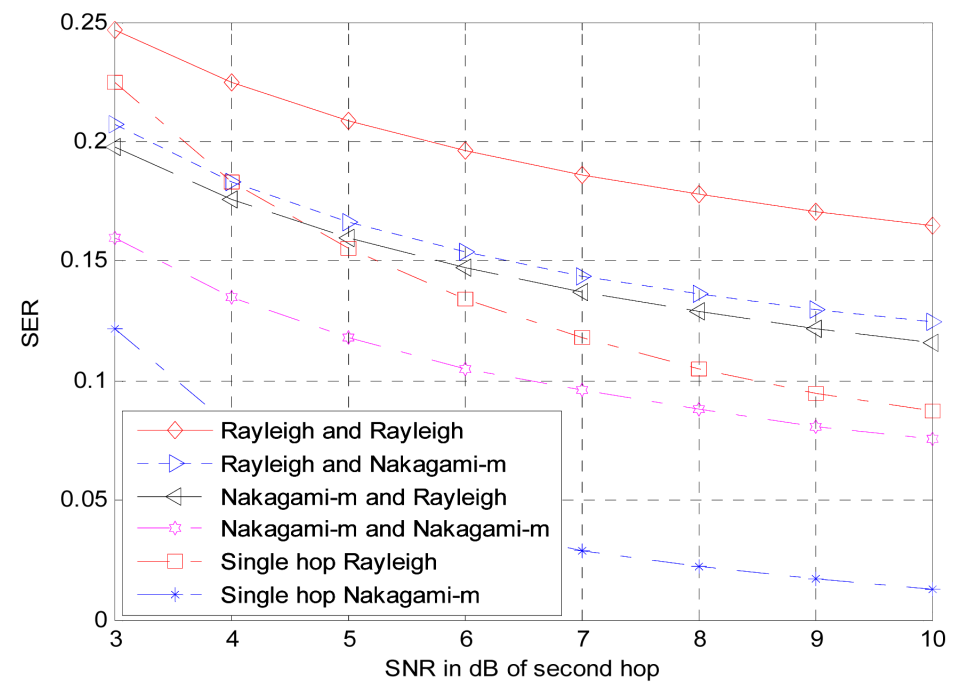

Figure 6. Variation of SER against SNR of $2^{\text {nd }}$ hop (strong $1^{\text {st }}$ hop).

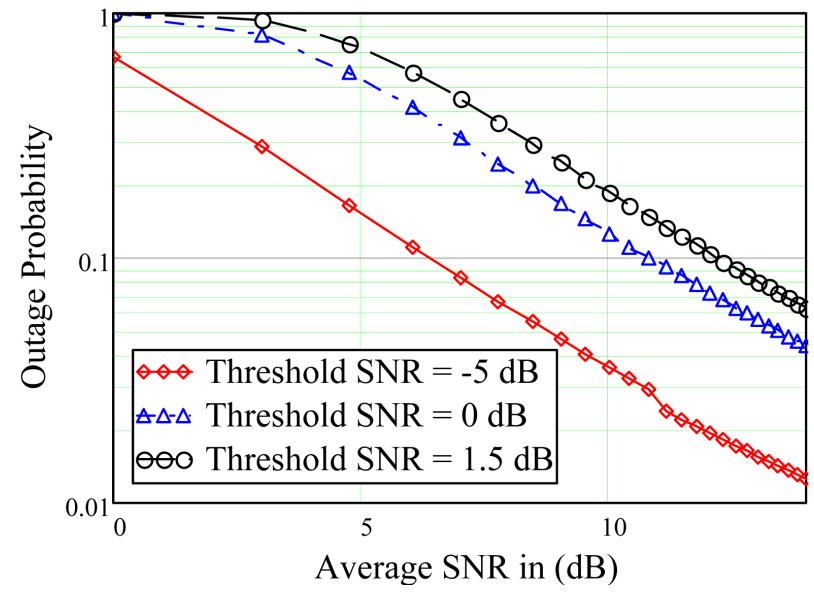

Figure 7. Variation of outage probability against average SNR ( $2^{\text {nd }}$ hop). 


\section{Conclusion}

In this paper we deal with the performance of 2-hop wireless link under asymmetric fading environment. Here, we have implemented the analytical model of asymmetric fading in a simpler way. We have combined the pdf of fading and the environment of AWGN to get the SER and Outage probability under asymmetric fading condition. Major applications of such network lie in: wireless ad-hoc network, wireless sensor network and virtual MIMO of 5G network. Here entire analysis is done under single antenna case. The work can be enhanced with multiple antennas on transmitting or receiving end; even on both sides. In this case we can apply the technique of TAS (transmitting antenna selection) on transmitting side or combining scheme on receiving side. Still we can apply zero forcing equalization technique or match filter on receiving end to improve the received SNR.

\section{References}

[1] Rankov, B. and Wittned, A. (2007) Spectral Efficient Protocols for Half-Duplex Fading Relay Channels. IEEE Journal on Selected Areas in Communications, 25, 379-389. http://dx.doi.org/10.1109/JSAC.2007.070213

[2] Hasna, M.O. and Alouini, M.-S. (2003) End-to-End Performance of Transmission Systems with Relays over Rayleigh Fading Channels. IEEE Transactions on Wireless Communications, 2, 1126-1131. http://dx.doi.org/10.1109/TWC.2003.819030

[3] Hasna, M.O. and Alouini, M.-S. (2004) Performance Study of Dual-Hop Transmissions with Fixed Gain Relays. IEEE Transactions on Wireless Communications, 3, 1963-1968. http://dx.doi.org/10.1109/TWC.2004.837470

[4] Chaudary, Muhammad Hasanain and Nandana Rajatheva, "Performance Analysis of Analog Network Coding Based Two-Way Amplify-and-Forward System in Mixed Rician and Nakagami-m Fading Environment. EURASIP Journal on Wireless Communications and Networking, 2012, 209. http://dx.doi.org/10.1186/1687-1499-2012-209

[5] Nadia, A., Chowdhuray, A.R., Islam, Md.I., Hossain, Md.S. and Amin, M.R. (2013) Performance Evaluation of TwoHop Wireless Link under Nakagami-m Fading. International Journal of Advanced Computer Science and Applications (IJACSA), 4, 142-146.

[6] Gurung, A.K., Al-Qahtani, F.S., Hussain, Z.M. and Alnuwarii, H. (2010) Performance Analysis of Amplify-Forward Relay in Mixed Nakagami- $m$ and Rician Fading Channels. International Conference on Advanced Technologies for Communications, HO Chi Minh City, 20-22 Oct. 2010, 321-326.

[7] Suraweera, H.A., Louie, R.H.Y., Li, Y.H., Karagiannidis, G.K. and Vucetic, B. (2009) Two Hop Amplify-and-Forward Transmission in Mixed Rayleigh and Rician Fading Channels. IEEE Communications Letters, 13, 227-229. http://dx.doi.org/10.1109/LCOMM.2009.081943

[8] Kapucu, N., Bilim, M., Develi, I. and Kabalci, Y. (2015) Performance of Two-Hop Relay Assisted Decode-andForward Transmission under Mixed Fading Environments. Elektronika ir Elektrotechnika, 21, 60-63.

[9] Yilmaz, A. and Kucur, O. (2011) Performance of Transmit Antenna Selection and Maximal-Ratio Combining in Dual Hop Amplify-and-Forward Relay Network over Nakagami-m Fading Channels. Wireless Personal Communications, 67, 485-503. http://dx.doi.org/10.1007/s11277-011-0391-7

[10] Chen, S., Wang, W., Zhang, X. and Zhao, D. (2009) Performance Amplify-and-Forward MIMO Relay Channels with Transmit Antenna Selection and Maximal Ratio Combining. IEEE Wireless Communications and Networking Conference (WCNC'09), Budapest, 5-8 April 2009, 1-6.

[11] Zedini, E., Ansari, I.S. and Alouini, M.-S. (2015) Performance Analysis of Mixed Nakagami-m and Gamma-Gamma Dual-Hop FSO Transmission Systems. IEEE Photonics Journal, 7, Article \#: 7900120. http://dx.doi.org/10.1109/JPHOT.2014.2381657

[12] Olabiyi, O. and Annamalai, A. (2012) Efficient Symbol Error Rate Analysis of Cooperative Non-Regenerative Relay Systems over Generalized Fading Channels. International Journal of Wireless \& Mobile Networks (IJWMN), 4, 1-20.

[13] Elkashlan, M., Yeoh, P.L., Yang, N., Duong, T.Q. and Leung, C. (2012) A Comparison of Two MIMO Relaying Protocols in Nakagami-m Fading. IEEE Transactions on Vehicular Technology, 61, 1416-1422. http://dx.doi.org/10.1109/TVT.2012.2185259 